\title{
Magnetic and microstructural analysis of palladium nanoparticles with different capping systems
}

\author{
R. Litrán,,${ }^{1 *}$ B. Sampedro, ${ }^{2}$ T. C. Rojas, ${ }^{1}$ M. Multigner, ${ }^{2}$ J. C. Sánchez-López, ${ }^{1}$ P. Crespo, ${ }^{2}$ C. López-Cartes,${ }^{1}$ \\ M. A. García, ${ }^{2}$ A. Hernando, ${ }^{2}$ and A. Fernández ${ }^{1}$ \\ ${ }^{1}$ Instituto de Ciencia de Materiales de Sevilla, CSIC-Univ.Sevilla, Américo Vespucio nr.49, 41092-Sevilla, Spain \\ ${ }^{2}$ Instituto de Magnetismo Aplicado (RENFE-UCM-CSIC), P.O. Box 155, 28230 Las Rozas, Madrid, Spain \\ and Departamento de Física de Materiales, Universidad Complutense, Madrid, Spain
}

(Received 20 May 2005; revised manuscript received 20 December 2005; published 3 February 2006)

\begin{abstract}
Palladium nanoparticles capped with different protective systems in a size range between 1.2 and $2.4 \mathrm{~nm}$ have been obtained by varying the preparation chemical method. Magnetization curves for all the samples show hysteresis loops, evidencing a ferromagnetic or a permanent magnetism in the nanoparticles. The microstructure of the nanoparticles has been analyzed by x-ray absorption and transmission electron microscopy. The nature of the magnetic behavior found for all these Pd nanoparticles (NPs) is different depending on their sizes and structural features and is explained on the basis of two different suggested mechanisms. The particles protected by means of a surfactant (tetralkylammonium salts), present a ferromagnetic order related to the factors increasing the density of states just below the Fermi level. Whereas, when the nanoparticles are stabilized by covalent bonds with protective species (thiol derivatized alkane chains or surface oxidized Pd $\mathrm{NPs}$ ), the increase of the $4 d$ density of holes, localized by the bonded atoms ( $\mathrm{S}$ or $\mathrm{O}$ ), is giving rise to the observed ferromagneticlike behavior.
\end{abstract}

DOI: 10.1103/PhysRevB.73.054404

PACS number(s): 75.75.+a, 61.46.-w, 87.64.Fb, 68.37.Lp

\section{INTRODUCTION}

Metallic nanoparticles (NPs) are extensively studied since they exhibit novel electronic, optical, and magnetic properties. In particular, the study of magnetism in clusters of palladium and in general $4 d$ elements has attracted special attention as these elements are nonmagnetic in bulk. ${ }^{1,2}$ Bulk palladium (fcc structure) presents a high paramagnetic susceptibility value, and it is close to fulfilling the Stoner criterion of magnetism, $\mathrm{N}\left(\mathrm{E}_{\mathrm{F}}\right) \mathrm{I}>1$, where $\mathrm{N}\left(\mathrm{E}_{\mathrm{F}}\right)$ is the density of states just below the Fermi level $\left(\mathrm{E}_{\mathrm{F}}\right)$, and I stands for the Stoner parameter (typically $0.71 \mathrm{eV}$ for $\mathrm{Pd}$ ). ${ }^{3}$ Accordingly, the factors that can affect the onset of ferromagnetism in Pd are those increasing $\mathrm{N}\left(\mathrm{E}_{\mathrm{F}}\right)$ in order to accomplish the Stoner criterion. $^{4}$

Experiments carried out by Taniyama et al. ${ }^{4}$ have evidenced the appearance of magnetic moment in Pd clusters with average radius below $7 \mathrm{~nm}$. Due to the strong surface energy anisotropy, these clusters of Pd exhibit a noncrystallographic icosahedral structure instead of the typical bulk fcc symmetry, which has been identified as the origin of this ferromagnetism. ${ }^{5}$ No size threshold has been demonstrated for this phenomena. Taniyama et al. ${ }^{5}$ found an increase of the ferromagnetic susceptibility by decreasing the particle size from 9.9 down to $5.9 \mathrm{~nm}$ of mean radius, however, experiments carried out by Cox et al. ${ }^{6}$ have not shown evidence of ferromagnetism on 13-150 atoms Pd clusters. Also hcp palladium has been recently shown to exhibit ferromagnetic behavior. ${ }^{7}$ In addition to that we have recently reported the ferromagnetic order in alkylammonium protected Pd NPs of $2.4 \mathrm{~nm}$. Local symmetry changes at twin boundaries, as well as surface anisotropy induced by reduction in coordination number were proposed as factors that could contribute to this magnetic behavior. ${ }^{1}$

On the other hand, we have also reported permanent magnetism, in small thiol capped gold ${ }^{8}$ clusters that cannot be explained by the above-mentioned criterion. In such a system, the large amount of sulfur atoms covalently bonded to the metal cluster, tend to induce " $d$ hole localization" in the metal core, due to charge transfer processes. Permanent magnetism up to room temperature was observed. ${ }^{8}$

In the present work, we have studied in a comparative way the microstructure and magnetic behavior of Pd NPs prepared with different capping environments. On one side weak interacting dipole molecules like tetralkylammonium $\left(\mathrm{R}_{4} \mathrm{~N}^{+} \mathrm{X}^{-}\right)$salts have been investigated also varying the length of the alkyl chains (what also produces a different degree of surface oxidation of the NPs). On the other side alkane-thiol molecules covalently bonded to palladium atoms in the clusters have been investigated. In this case two different particle sizes of the palladium nanoparticles have been considered.

Combining the results obtained by chemical analysis with transmission electron microscopy (TEM) and extended x-ray absorption fine structure (EXAFS), an exhaustive characterization at the microstructural level has been performed. The magnetic behavior will be analyzed on the basis of the structural information.

\section{EXPERIMENT}

Palladium nanoparticles (NPs) have been obtained by chemical reduction and stabilized by means of either a surfactant, or by promoting covalent bonds between the metallic surface and alkanethiolate chains.

In the first case, Pd NPs have been prepared by a redoxcontrolled size-selective method, using tetralkylammonium salts $\left(\mathrm{R}_{4} \mathrm{~N}^{+} \mathrm{X}^{-}\right)$as surfactant in tetrahydrofuran (THF). For preparing sample $\mathrm{PdNR}_{4}\left(\mathrm{C}_{4}\right)$, a solution of $\mathrm{Pd}\left(\mathrm{NO}_{3}\right)_{2}$ from Aldrich (99\%) (0.02 g in $25 \mathrm{ml}$ of THF) was used as a palladium precursor in an excess of tetrabutylammonium ac- 
etate $(0.1 \mathrm{~g}$ in $2.5 \mathrm{ml}$ of THF). The resulting mixture was heated to reflux and kept at $343 \mathrm{~K}$ for $30 \mathrm{~min}$. Nitrate is displaced by the more effective acetate ligand, which is followed by the reduction of the $\mathrm{Pd}^{2+}$ by electron-transfer, giving rise to $\mathrm{Pd}^{0}$-stabilized clusters. ${ }^{9}$ Finally the solution was cooled to room temperature and dried at ambient air. The $\mathrm{Pd}-\mathrm{NR}_{4}(\mathrm{C} 12)$ sample was synthetized by the same method but using a solution of $0.008 \mathrm{~g}$ of $\mathrm{Pd}\left(\mathrm{NO}_{3}\right)_{2}$ in $10 \mathrm{ml}$ of THF, tetradodecylammonium nitrate as surfactant $(0.1 \mathrm{~g})$ and sodium acetate as the reducing species $(0.01 \mathrm{~g})$ both mixed using $1 \mathrm{ml}$ of THF.

In the second case alkanethiolate-capped palladium NPs have been obtained by a liquid-liquid phase reduction at room temperature, based on the Brust method, ${ }^{10}$ varying the molar ratio of $\mathrm{Pd}$ :thiol. In the first step, $\mathrm{Pd}^{2+}$ is transferred from an aqueous solution, containing $\mathrm{Pd}\left(\mathrm{NO}_{3}\right)_{2}$ from Aldrich (99\%) $(0.05 \mathrm{~g}$ in $10 \mathrm{ml}$ of $\mathrm{HCl} 0.5 \mathrm{~N})$, to degassed and dried toluene using tetraoctylammonium bromide ( $98 \%$, Aldrich) ( $0.55 \mathrm{~g}$ in $20 \mathrm{ml}$ of toluene) as the phase-transfer agent. The mixture is strongly stirred for $30 \mathrm{~min}$. Once the aqueous phase is removed, $0.1 \mathrm{ml}$ of dodecanethiol $(0.05 \mathrm{ml}$ for $\mathrm{Pd}-$ SR2 sample) is added to the organic phase under strong stirring and kept for $15 \mathrm{~min}$. Then the Pd precursor is reduced, using an aqueous solution of $\mathrm{NaBH}_{4}(99 \%$, Aldrich) $(0.1 \mathrm{~g}$ in $5 \mathrm{ml}$ of $\mathrm{H}_{2} \mathrm{O}$ ) as the reducing agent. The presence of dodecanethiol leads to the formation of $\mathrm{Pd}-\mathrm{S}$ bonds and isolates the metal clusters, preventing agglomeration. Subsequently, the organic phase was separated from the aqueous phase and the toluene removed under reduced pressure by means of a rotary evaporator. Pd NPs were precipitated with methanol and filtered. The Pd:thiol ratio is one of the factors controlling the final size of obtained nanoclusters. ${ }^{11}$ With the aim to obtain palladium nanoparticles with different average sizes, reactions have been carried out for $\mathrm{Pd}$ :thiol molar ratios ranging from 1:2 (sample referred as Pd-SR1) to 1:1 (PdSR2 sample).

All the reduction reactions during preparation of the different NPs were carried out under $\mathrm{N}_{2}$ atmosphere to minimize oxidation. Ulterior washing, drying, storage, and transfer to superconducting quantum interference device (SQUID), TEM, and EXAFS were carried out in ambient air atmosphere. Only for storage the samples were placed in a dessicator.

The chemical composition of the samples has been evaluated by ICP (inductively coupled plasma-atomic emission spectrometry) analysis, elemental chemical analysis, and energy dispersive $\mathrm{x}$-ray analysis (EDX) at the transmission electron microscope. The level of Fe impurities in all samples was found to be between 30 and 70 ppm as determined by ICP.

TEM analysis was carried out in a Philips CM200 microscope working at $200 \mathrm{kV}$ with a $\mathrm{LaB}_{6}$ filament. Particle size distribution histograms were measured using an image analyzer.

X-ray absorption spectra (XAS) for Pd NPs were recorded in transmission mode at the beam line BM29 in the ESRF storage ring in samples supported on $\mathrm{BN}$ pressed pellets. Spectra were recorded at the Pd K edge at ca. 24,35 keV. The EXAFS oscillations were quantitatively analyzed with the software package developed by Bonin et al. ${ }^{12}$ The coordina- tion numbers $(\mathrm{N})$, distances $(\mathrm{R})$, and Debye-Waller factors $(\sigma)$ were extracted by a least-squares fitting procedure that uses the theoretical phases and amplitudes proposed by Rehr and co-workers. ${ }^{13}$ These parameters have been previously calibrated with a $\mathrm{Pd}$ foil reference.

Magnetic measurements have been performed using a Quantum Design SQUID magnetometer. The diamagnetic contribution corresponding to the sample holder system has been previously measured. After subtracting this contribution from the total magnetization, values corresponding to Pd can be directly obtained. The samples after preparation and purification were stored in a dessicator in the form of dried powder. For SQUID measurements the samples were then measured as powders, slightly compacted inside the sample holder.

\section{RESULTS AND DISCUSSION}

\section{A. Microstructural and chemical characterization of NPs}

Figure 1 shows the transmission electron micrographs for the four samples, as well as their corresponding particle size distribution histograms. The NPs stabilized by means of a surfactant, Pd-NR 4 (C4) [Fig. 1(a)] and Pd-NR 4 (C12) [Fig. 1(b)], show similar average particle sizes, 2.4 and $2.1 \mathrm{~nm}$, respectively, as well as narrow particle size distributions. The general view of Pd-SR1 sample [Fig. 1(c)] evidences the presence of very small clusters, approximately $1.2 \mathrm{~nm}$ in size, embedded in an amorphous matrix, while for the $\mathrm{Pd}$ SR2 NPs [Fig. 1(d)] isolated nanoparticles of $2.3 \mathrm{~nm}$ average size can be easily distinguished. Although the average diameter for Pd-SR2 NPs, $2.3 \mathrm{~nm}$, is approximately the same than for particles prepared with alkylammoniun salts, the covalent bonds with the alkanethiolate chains allows the stabilization of isolated nanoparticles while the alkylammonium protected clusters tend to be aggregated.

Table I shows the size and chemical composition data obtained for the different Pd samples. From ICP analysis, around $10 \%$ of palladium was found for $\mathrm{Pd}_{-} \mathrm{NR}_{4}(\mathrm{C} 4)$ and Pd-NR $4(\mathrm{C} 12)$ samples, and $18 \%$ and $26 \%$ for Pd-SR 1 and Pd-SR2, respectively. The percentages of $\mathrm{S}$ and $\mathrm{N}$ have been obtained from elemental chemical analysis. For alkanethiolate capped NPs, in which covalent bonds are formed, the atomic ratio of $\mathrm{S} / \mathrm{Pd}$ could give information on the number of chains linked to $\mathrm{Pd}$ atoms. In the case of alkylammonium protected NPs, the number of protective molecules directly in contact with the particle surface cannot be deduced from the chemical analysis due to multilayered arrangement of dipole molecules around the NPs.

It has been previously shown ${ }^{14}$ that for Pd NPs protected by alkanethiolate molecules, the surface of the Pd core is sulfurized, to form a mixed Pd-S layer underneath the thiolate monolayer. This effect was also proposed in our previous papers for Au NPs functionalized by alkanethiolates. 8,15 In the present work according to the data in Table I, the total number of $\mathrm{Pd}$ atoms for the Pd-SR1 sample has been estimated to be around 43, with $98 \%$ located at the surface (in fact, this number represents the number of $\mathrm{Pd}$ atoms with deficient coordination shell for a pure palladium cluster of 

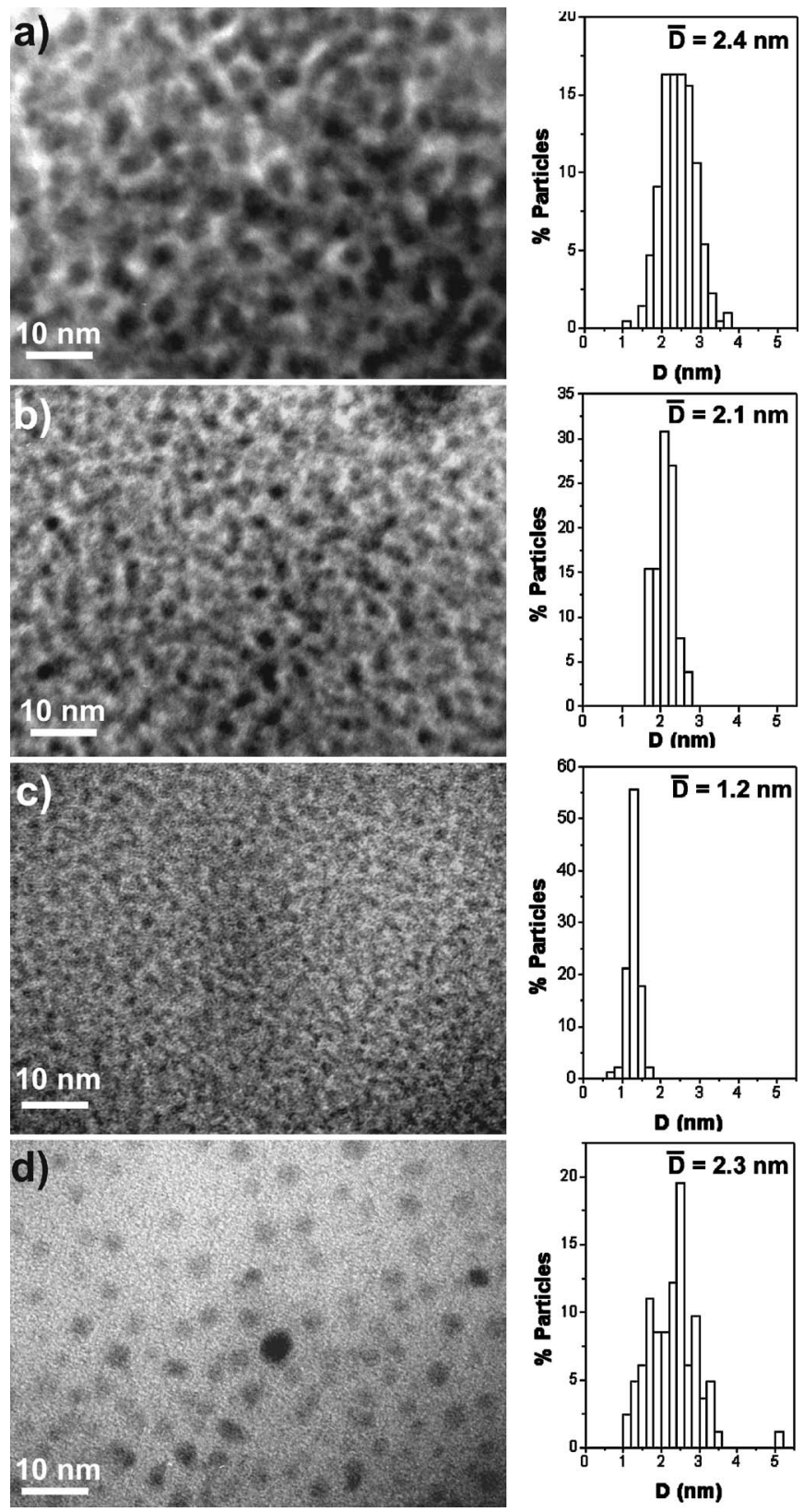

FIG. 1. Transmission electron micrographs and their corresponding particle size distribution histograms of the Pd NPs under study: (a) Pd-NR ${ }_{4}(\mathrm{C} 4)$, (b) $\mathrm{Pd}-\mathrm{NR}_{4}(\mathrm{C} 12)$, (c) Pd-SR1, and (d) Pd-SR2.

the corresponding particle size ${ }^{15}$ ). Even if each atom at the surface had a bond with an alkanethiolate chain, the atomic ratio $\mathrm{S} / \mathrm{Pd}$ would reach 0.98 . However, the experimental value obtained by chemical analysis for this sample is 1.8 , which clearly exceeds the theoretical calculations. Taking into account the same considerations for the Pd-SR2 sample, the atomic ratio $\mathrm{S} / \mathrm{Pd}$ would be 0.56 , with an experimental value of 1.0. All these data support the Pd-S depletion layer. This phenomenon is especially significant for the small 1.2 $\mathrm{nm}$ clusters, in which the final number of $\mathrm{Pd}-\mathrm{S}$ bonds is considerably increased, and the remaining pure Pd cluster may contain a few Pd atoms. Figure 2(a) represents the radial distribution functions (not corrected for phase shifts), ob- tained by Fourier transformation (FT) of the EXAFS oscillations raw data, at the Pd K edge for the Pd-SR1 sample as compared to a Pd foil reference sample (k range 3-13 $\AA$ ). Fitting procedure analysis was done assuming first ( $\mathrm{Pd}-\mathrm{S})$ and second (Pd-Pd) coordination shells. Coordination numbers of $2.7 \pm 0.5$ for Pd-S and $1.2 \pm 0.2$ for Pd-Pd coordination shells were obtained together with $\mathrm{Pd}-\mathrm{S}$ and $\mathrm{Pd}-\mathrm{Pd}$ bond distances of $2.32 \pm 0.01$ and $2.76 \pm 0.1 \AA$, respectively. A Pd-Pd bond distance of $2.71 \pm 0.01 \AA$ has been obtained for the reference $\mathrm{Pd}$ foil whereas the reported $\mathrm{Pd}-\mathrm{S}$ bond distance for a bulk palladium sulphide pellet was $2.33 \AA .{ }^{16}$ The very small Pd-Pd coordination number does not correspond with $1.2 \mathrm{~nm}$ size pure Pd clusters. ${ }^{16}$ In addition to that the high Pd-S coordination number is in agreement with the formation of a depletion Pd-S shell in the small $1.2 \mathrm{~nm}$ size clusters. The size of the metallic fcc Pd cluster is estimated to be smaller than $1 \mathrm{~nm}$ and therefore the number of $\mathrm{Pd}$ metallic atoms is much less than 43 .

Regarding the alkylammonium protected NPs, Fig. 2(b) is showing the radial distribution functions (not corrected for phase shifts), obtained by Fourier transformation (FT) of the EXAFS oscillations raw data, at the $\mathrm{Pd} \mathrm{K}$ edge for the $\mathrm{Pd}-\mathrm{NR}_{4}(\mathrm{C} 4)$ and the $\mathrm{Pd}-\mathrm{NR}_{4}(\mathrm{C} 12)$ samples as compared to a Pd foil reference sample (k range 3-10 A). The differences in the Pd foil curves in Figs. 2(a) and 2(b) are due to the different $\mathrm{k}$ range used for the Fourier transform calculations. According to the FT curves in Fig. 2(b), the Pd atoms in the here studied alkylammonium protected NPs show a first coordination shell at a short distance $(2.07 \pm 0.01 \AA$ after fitting) that can correspond to $\mathrm{Pd}-\mathrm{C}$ or $\mathrm{P}-\mathrm{O}$ bonds. A second coordination shell is observed due to $\mathrm{Pd}-\mathrm{Pd}$ bonds at $2.67 \pm 0.01 \AA$ in good agreement with the value obtained for a Pd foil. The intensity of this peak is in agreement with the NPs sizes. The intensity of the first coordination shell is lower for $\mathrm{Pd}-\mathrm{NR}_{4}(\mathrm{C} 4)$ than for $\mathrm{Pd}-\mathrm{NR}_{4}(\mathrm{C} 12)$ although a similar particle size has been measured for both samples. In a previous paper Morow et al. ${ }^{17}$ have presented a detailed model of the protection shell of $\mathrm{N}(\mathrm{alkyl})_{4} \mathrm{C} 1$ stabilized colloidal particles. In this system chlorine atoms form an interstitial layer between the metal core and the surfactant shell. The interstitial chlorine ion is subjected to an attractive electrostatic force toward the $\mathrm{N}(\mathrm{alkyl})_{4}{ }^{+}$ion so that the position of the negative ion depends on the alkyl chain length. For very long alkyl chains steric impediments produce a less dense protection shell. The less effective protection of the longer alkyl chain molecules for oxygen diffusion during air storage produces in the $\mathrm{Pd}-\mathrm{NR}_{4}(\mathrm{C} 12)$ sample an increase in surface coordination (higher intensity of the first peak in the FT) probably due to a partial oxidation of the Pd surface.

Figure 3 shows the proposed models for the different capping/protection structures of the Pd NPs investigated in the present work. Based on the data obtained by both chemical as well as EXAFS analysis, we propose a core-shell microstructure for the Pd-SR NPs, which explains the excess of $\mathrm{S}$ atoms obtained. For sample Pd-SR1 the remaining metallic palladium cluster is very small. We have also proposed a small oxide shell surrounding the metal cluster in the case of the $\mathrm{Pd}-\mathrm{NR}_{4}(\mathrm{C} 12)$ sample, as compared to an almost pure metallic Pd nanoparticle in the case of the $\mathrm{Pd}_{-} \mathrm{NR}_{4}(\mathrm{C} 4)$ 
TABLE I. Size and chemical compositions results for the different Pd nanoparticles

\begin{tabular}{|c|c|c|c|c|c|c|c|}
\hline \multirow[b]{2}{*}{ Sample } & \multicolumn{3}{|c|}{$\%$ weight } & \multirow[b]{2}{*}{$\begin{array}{c}\mathrm{S} / \mathrm{Pd} \\
\text { (atomic ratio) }\end{array}$} & \multirow[b]{2}{*}{$\begin{array}{c}\text { Dm } \\
(\mathrm{nm})^{\mathrm{a}}\end{array}$} & \multirow[b]{2}{*}{$\begin{array}{l}\text { Total } \\
\mathrm{Nr}^{\mathrm{b}}\end{array}$} & \multirow[b]{2}{*}{$\% \mathrm{Nr}^{\mathrm{b}}$} \\
\hline & $\mathrm{Pd}$ & S & $\mathrm{N}$ & & & & \\
\hline $\mathrm{Pd}-\mathrm{NR}_{4}(\mathrm{C} 4)$ & 10 & & 5.5 & & $2.4 \pm 0.43$ & 369 & 55 \\
\hline $\mathrm{Pd}^{-\mathrm{NR}_{4}}(\mathrm{C} 12)$ & 9.8 & & 3.5 & & $2.1 \pm 0.26$ & 249 & 65 \\
\hline Pd-SR1 & 18 & 7.9 & & 1.8 & $1.2 \pm 0.15$ & 43 & 98 \\
\hline Pd-SR2 & 26 & 8.8 & & 1.0 & $2.3 \pm 0.64$ & 321 & 56 \\
\hline
\end{tabular}

${ }^{\mathrm{a}}$ Average particle size as calculated from particle size distribution histograms. The measured sizes by TEM includes the Pd metallic core and the mixed Pd-S layer (or Pd-O layer for oxidized Pd NPs, see description in text).

bTotal Nr: calculated number of atoms for a pure palladium cluster. $\% \mathrm{Nr}$ : calculated percentage of atoms located at the surface of a pure palladium cluster.

sample. In all cases the average particle sizes in Table I, as determined by TEM, correspond to the metallic core plus the depletion layer when present.

\section{B. Magnetic properties}

Figures 4-7 show the hysteresis loops measured at different temperatures for the four different samples, showing evidence of ferromagnetic order in all the cases. As we have previously reported, ${ }^{1}$ a clear ferromagnetic behavior with a Curie temperature above $300 \mathrm{~K}$ is observed for Pd-NR ${ }_{4}(\mathrm{C} 4)$ (Fig. 4). From the shape of the hysteresis loops obtained at different temperatures between 5 and $275 \mathrm{~K}$ it is inferred that particles with blocked magnetization contribute to the magnetization process. Coercivity decreases with temperature roughly linearly from $140 \mathrm{Oe}$ at $5 \mathrm{~K}$ down to zero at $340 \mathrm{~K}$. Hence, $340 \mathrm{~K}$ should rather correspond to a blocking temperature, $\mathrm{T}_{\mathrm{b}}$. By extrapolating at zero field the high field magnetization in Fig. 4, a value close to $0.02 \mathrm{emu} / \mathrm{g}$ is obtained. However, the magnetization reached at the high field branch of the loop is far from saturation as it is probably due to the coexistence of blocked ferromagnetic entities with superparamagnetic particles and paramagnetic atoms. ${ }^{1}$
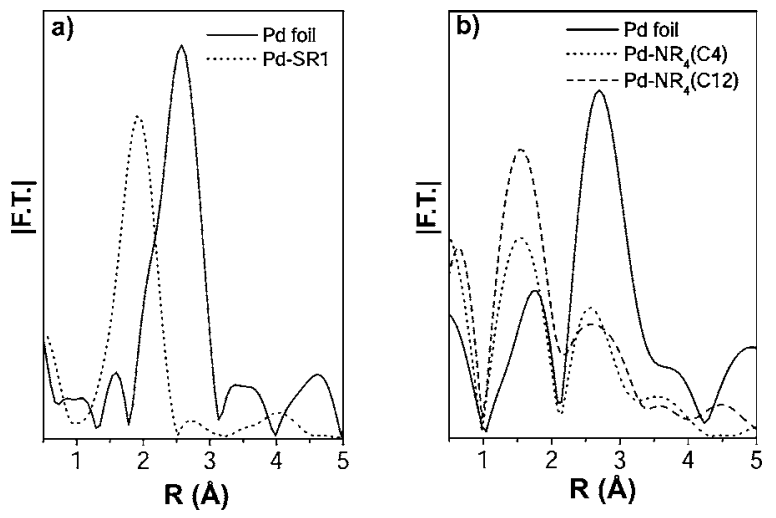

FIG. 2. Modulus of the Fourier transform at the Pd-K edge (K weighted, without phase correction) for (a) Pd-SR1 sample (k range 3-13 $\AA$ ) and (b) $\mathrm{Pd}-\mathrm{NR}_{4}(\mathrm{C} 4)$ and $\mathrm{Pd}_{-} \mathrm{NR}_{4}(\mathrm{C} 12)$ samples (k range 3-10 A) as compared to bulk palladium.
The same behavior is found for $\mathrm{Pd}-\mathrm{NR}_{4}(\mathrm{C} 12)$ for a temperature range between 100 and $275 \mathrm{~K}$ (Fig. 5), with magnetization and coercivity values similar to those obtained for $\mathrm{Pd}-\mathrm{NR}_{4}(\mathrm{C} 4)$. This analogous tendency is expected, since both samples are constituted by NPs similar in size and stabilized by the interactions with alkylammonium salts of different alkyl-chain length. However, while $\mathrm{Pd}-\mathrm{NR}_{4}(\mathrm{C} 4)$ keeps the same behavior for all the temperature range with variations only in the coercivity values, in the case of $\mathrm{Pd}-\mathrm{NR}_{4}(\mathrm{C} 12)$ the magnetization curve completely changes at $5 \mathrm{~K}$. A hysteresis loop, indicating a permanent magnetism, is

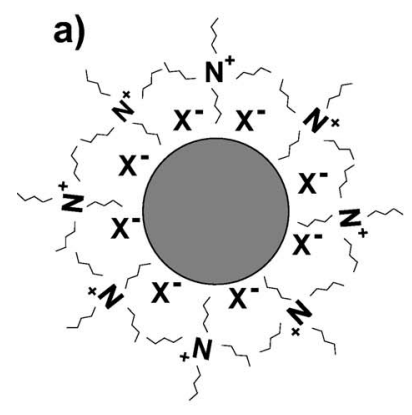

Pd core

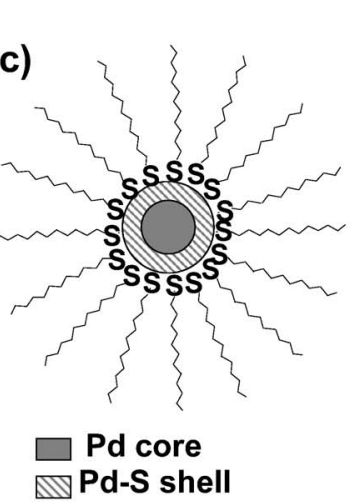

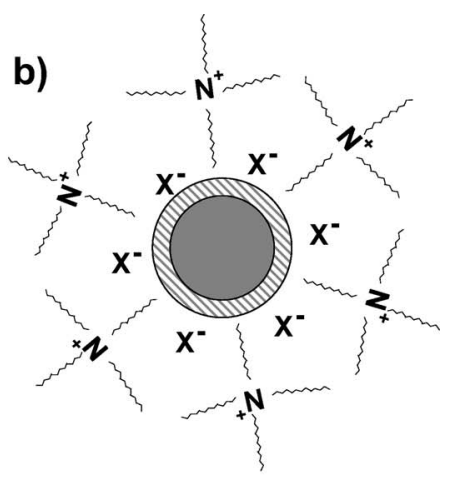

Pd core

Pd-O shell

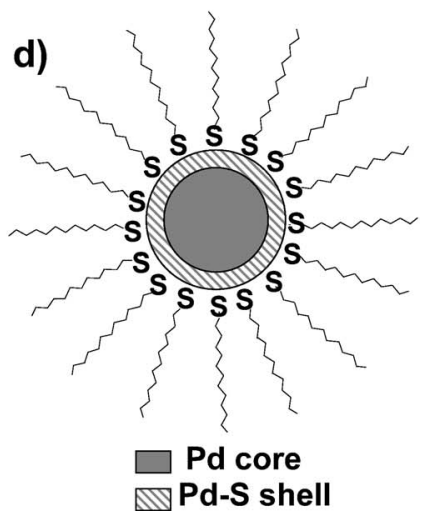

FIG. 3. Scheme of the proposed structures for (a) $\mathrm{Pd}_{-}-\mathrm{NR}_{4}(\mathrm{C} 4)$, (b) Pd-NR 4 (C12), (c) Pd-SR1, and (d) Pd-SR2. 


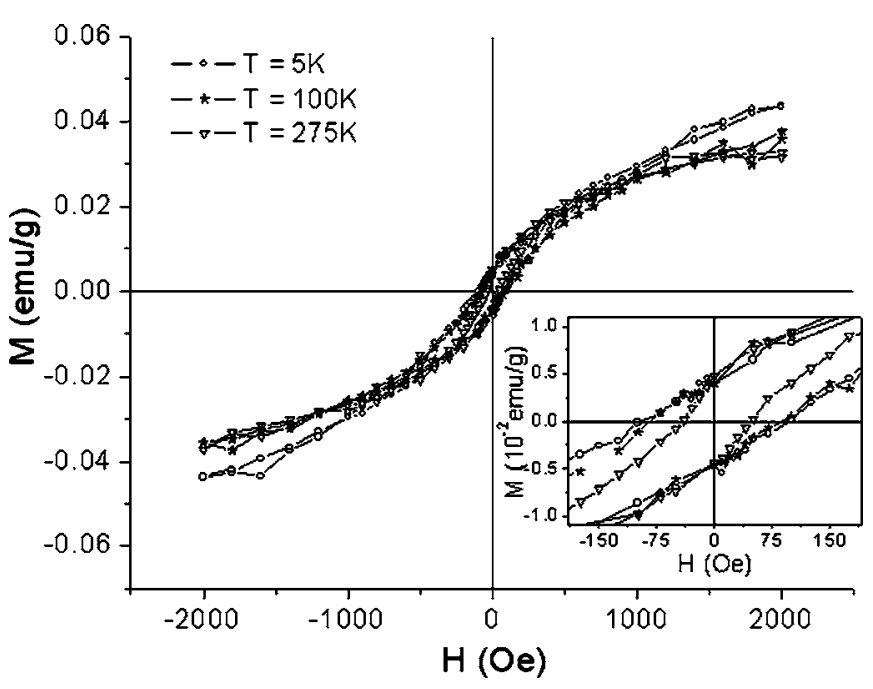

FIG. 4. Hysteresis loops at different temperatures for the Pd-NR 4 (C4) sample. The inset shows a more detailed view of the thermal coercivity response.

also found at this low temperature, but with coercivity lower than 40 Oe and the highest value of magnetization close to $0.2 \mathrm{emu} / \mathrm{g}$.

These magnetization values, found for $\mathrm{Pd}_{-} \mathrm{NR}_{4}(\mathrm{C} 12)$ at 5 $\mathrm{K}$, are similar to those obtained for all of the temperature range for the Pd-SR1 sample, in which there are covalent bonds between sulfur and palladium in the metal clusters (Fig. 6). In the case of the Pd-SR1 sample, since the hysteresis loops do not appreciably change with temperature, it is inferred that any possible superparamagnetic contribution is negligible. From these measurements it is also evidenced that there are no substantial changes in coercivity values from 5 to $300 \mathrm{~K}$, indicating that $\mathrm{T}_{\mathrm{B}}$ is much higher than $300 \mathrm{~K}$. The maximum values of magnetization also remain roughly constant, close to $0.18 \mathrm{emu} / \mathrm{g}$, one order of magnitude larger than the one observed for $\mathrm{Pd}-\mathrm{NR}_{4}(\mathrm{C} 4)$ and for $\mathrm{Pd}-\mathrm{NR}_{4}(\mathrm{C} 12)$ between 100 and $275 \mathrm{~K}$. Saturation value for

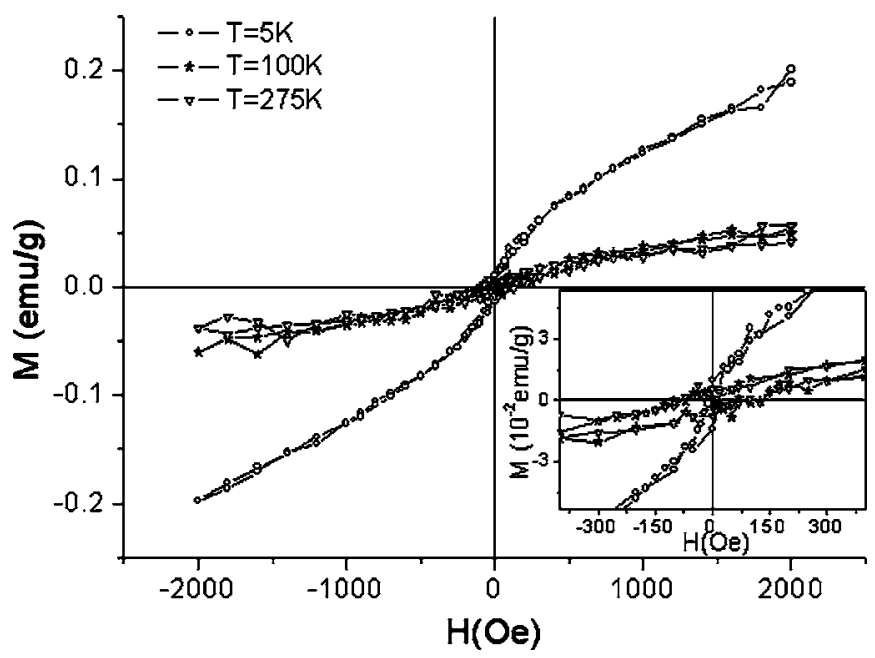

FIG. 5. Hysteresis loops at different temperatures for the $\mathrm{Pd}-\mathrm{NR}_{4}(\mathrm{C} 12)$ sample. The inset shows a more detailed view of the thermal coercivity response.

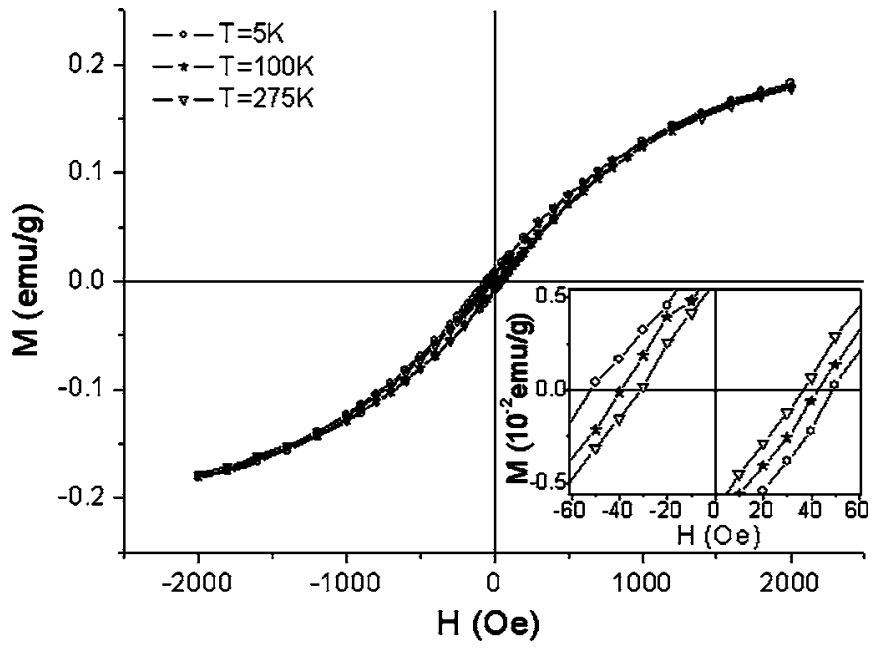

FIG. 6. Hysteresis loops at different temperatures for the Pd-SR1 sample. The inset shows a more detailed view of the thermal coercivity response.

magnetization is not reached at any temperature. The more surprising feature of the experimental results for this sample is the observation of coercivity at room temperature for such small metal cores. The behavior of the $\mathrm{Pd}-\mathrm{NR}_{4}(\mathrm{C} 12)$ sample at $5 \mathrm{~K}$, similar to the one observed for the Pd-SR1 sample, may be due to the surface oxidation of the palladium clusters leading to the formation of $\mathrm{Pd}-\mathrm{O}$ bonds that may produce similar effects to the ones produced by $\mathrm{Pd}-\mathrm{S}$ bonds at the surface of the thiol capped NPs.

The $2.3 \mathrm{~nm}$ alkanethiol capped palladium, Pd-SR2 sample, shows similar values of coercivity, but extremely lower magnetization than Pd-SR1 (Fig. 7). That sample also presents hysteresis loops; however, as opposed to the Pd-SR1 there is a Curie temperature close to $275 \mathrm{~K}$, at which the ferromagnetic order clearly disappears. In addition to that, the measurements obtained for this sample are very noisy due to the low magnetization values found for this sample.

Our results point out the existence of hysteresis loops in all cases. However, attending to the magnetization curves,

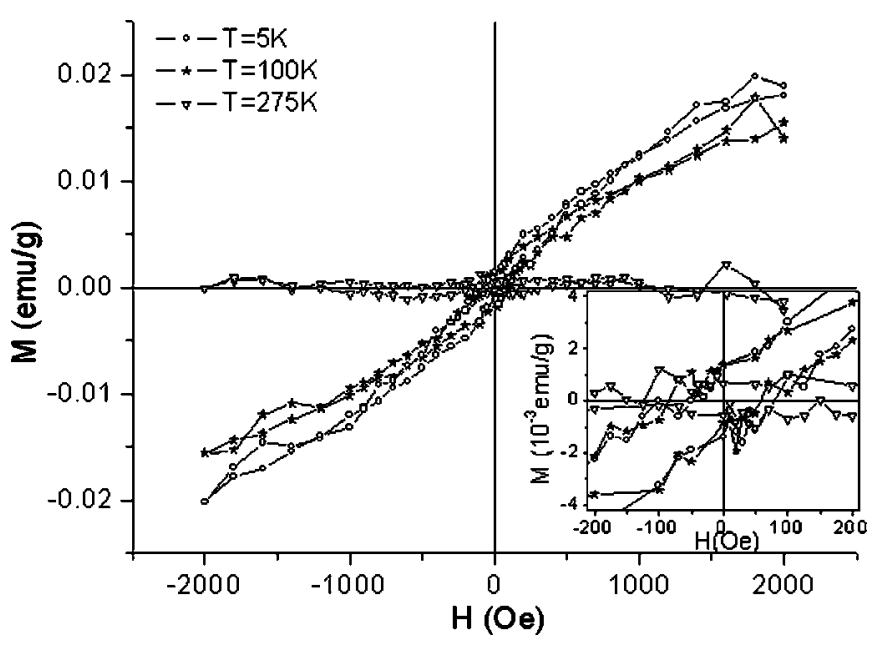

FIG. 7. Hysteresis loops at different temperatures for the Pd-SR2 sample. The inset shows a more detailed view of the thermal coercivity response. 
two main tendencies are observed, leading to magnetization values close to 0.02 or $0.2 \mathrm{emu} / \mathrm{g}$, respectively. These two behaviors are probably originated by two different phenomena, depending on Pd NPs particle size and/or on the capping molecule.

Factors that can affect the onset of ferromagnetism in late $4 \mathrm{~d}$ transition metals in general and $\mathrm{Pd}$ in particular, are those increasing the density of states just below the Fermi level allowing fulfillment of the Stoner criterion for ferromagnetism. Reduced coordination number and surface anisotropy together with local symmetry changes or lattice expansion can be proposed as possible factors to induce the ferromagnetic behavior in palladium NPs around $2 \mathrm{~nm}$ in size. Some of these factors can play a role in our alkylammonium protected NPs, where there are not covalent bonds between the metal core and the protective surfactant. Specifically, surface anisotropy induced by reduction in the coordination number as well as changes in the local symmetry at the twin boundaries have been proposed in a previous paper ${ }^{1}$ to account for the presence of ferromagnetism in the $\mathrm{Pd}-\mathrm{NR}_{4}(\mathrm{C} 4)$ sample. Surface atoms are located in a non-fcc symmetry and consequently, an extremely high percentage of them $[55 \%$ for $\mathrm{Pd}-\mathrm{NR}_{4}(\mathrm{C} 4)$ and $65 \%$ for $\left.\mathrm{Pd}-\mathrm{NR}_{4}(\mathrm{C} 12)\right]$ could be invoked as a possible cause of the onset of ferromagnetism. The eventual increase of $\mathrm{N}\left(\mathrm{E}_{\mathrm{F}}\right)$ at the cluster surface due to the enhancement of electron localization, as well as the effect produced by the electrostatic interaction with dipole surfactant molecules, may contribute to the appearance of a permanent magnetic moment and high local magnetic anisotropy. Also the enhancement of orbital magnetic moments at the surface $\mathrm{Pd}$ atoms can contribute to the total magnetic moment. In the case of the $\mathrm{Pd}-\mathrm{NR}_{4}(\mathrm{C} 12)$ sample the presence of oxide surface species can introduce new effects and explains the higher magnetization values at $5 \mathrm{~K}$ as it will be discussed below.

In addition to surface effects, local deviation from cubic symmetry can also occur near twin boundaries inside a nanoparticle. High resolution electron microscopy (HREM) was used in the previous publication ${ }^{1}$ to analyze the presence of such twin boundaries in the $\mathrm{Pd}-\mathrm{NR}_{4}(\mathrm{C} 4)$ sample. The relevant issue is that the atoms located at twin boundaries show a noncubic local field symmetry. The d-atomic level does not split into $e_{g}$ and $t_{2 g}$ sublevels giving rise to a drastic narrowing of the $4 d$ band sufficient for the Stoner criterion to be met. The strong surface energy anisotropy acts as the driving force for twinning in small Pd clusters. Therefore the small fraction of atoms located at the vicinity of the twin boundaries can contribute to the onset of ferromagnetism experimentally observed in these systems. In addition to that the partly sintered particles observed in sample $\mathrm{Pd}-\mathrm{NR}_{4}\left(\mathrm{C}_{4}\right)$ [see Fig. 1(a)], may favor the formation and stabilization of twin boundaries in this sample.

The situation completely changes when the metal nanoclusters are covalently bonded to protective species, such as thiol chains. In these systems the effect of interactions with capping molecules become stronger than the rest of the referred factors (i.e., surface-induced anisotropy or twining). This phenomenon is especially noticeable for the Pd-RS1 sample, where the above-mentioned factors, increasing the density of states just below the Fermi level, are not probably stabilized due to the extremely small size of the metal clusters (complete absence of twin boundaries). Almost all the $\mathrm{Pd}$ atoms (less than 50), forming the core are in fact surface atoms linked to $\mathrm{S}$ atoms. It is important to consider that, for such a small number of atoms, the presence of permanent magnetism does not necessarily imply the existence of ferromagnetic order but only reveals strong magnetic anisotropy. This permanent magnetism could be explained considering it is likely that the thiol bond increases the density of holes, $\xi$, at the $4 \mathrm{~d}$ residual band of the Pd cluster giving rise to an increase of the magnetic moment. These holes induced by bonding are localized nearby the region of overlapping $4 \mathrm{~d}-\mathrm{Pd}$ and $2 \mathrm{p}-\mathrm{S}$ charges. Therefore the permanent magnetism reported above does not seem to be related with the metallic ferromagnetic instability of bulk Pd derived from its high density of states near the Fermi level. It is probably associated - or at least enhanced-with the chemical bonding to $\mathrm{S}$ atoms giving rise to localized charge transfer and to the strong anisotropy of the $\mathrm{Pd}$ atoms submitted simultaneously to $\mathrm{Pd}-\mathrm{Pd}$ and $\mathrm{Pd}-\mathrm{S}$ bonds. It is a magnetism highly anisotropic and largely orbital in character. ${ }^{18,19} \mathrm{~A}$ similar mechanism has also been proposed by us for small thiol capped gold NPs. ${ }^{8}$

The increasing density of holes at the $4 \mathrm{~d}$ residual band of Pd clusters is less efficient in the case of the Pd-SR2 system, due to the larger size of the metal clusters and consequently the lower fraction of $\mathrm{Pd}-\mathrm{S}$ bonds allowed in the depletion shell.

The EXAFS analysis for sample $\mathrm{Pd}-\mathrm{NR}_{4}(\mathrm{C} 12)$ reveals the existence of a bigger fraction of palladium oxide in the sample as compared to $\mathrm{Pd}-\mathrm{NR}_{4}(\mathrm{C} 4)$. This partial oxidation of sample $\mathrm{Pd}-\mathrm{NR}_{4}(\mathrm{C} 12)$ leads to the formation of $\mathrm{Pd}-\mathrm{O}$ bonds, which in a similar way to $\mathrm{Pd}-\mathrm{S}$ bonds, produce an increase in the density of holes at the $4 \mathrm{~d}$ residual band of $\mathrm{Pd}$, inducing a permanent magnetism observed only at $5 \mathrm{~K}$ for this sample. The relatively large atomic weight of $S$ when compared with $\mathrm{O}$ accounts for a larger $\mathrm{Pd}-\mathrm{S}$ spin-orbit coupling strength that makes the sulfur species more effective for frozen magnetic moments. When the increase in the density of holes is promoted by the $\mathrm{O}$ bonds instead of $\mathrm{S}$, the low temperature of $5 \mathrm{~K}$ is necessary to get an effective fixation of moments, whereas at higher temperatures the effects increasing the density of states near the Fermi level are prevalent.

As the NPs are protected by the capping molecules, possible interparticle interactions can be only of magnetostatic nature. Even if the particles are aggregated, the average distance between Pd NPs is determined by the length of the thiol-alkyl chain $(1.7 \mathrm{~nm})$ or the size of the tetralkylammonium salt protective molecule [approximately $3.0 \mathrm{~nm}$ for $\mathrm{NR}_{4}(\mathrm{C} 12)$ and $1.0 \mathrm{~nm}$ for $\left.\mathrm{NR}_{4}(\mathrm{C} 4)\right]$. As the permanent magnetic moment of each particle is very low, the magnetic field acting on a NP due to a single neighbor NP is also very low; therefore the influence of the stray field can be neglected.

In summary, the magnetism observed in these Pd NPs is explained on the basis of two different phenomena. The particles protected by means of a surfactant and without surface oxidation, present a ferromagnetic order related to the factors increasing the density of states just below the Fermi level. 
Whereas, when nanoparticles are stabilized by covalent bonds with protective species a depletion shell structure is promoted, the atoms depleting the NP are able to increase the $4 \mathrm{~d}$ density of holes at the Pd site and to localize a magnetic moment, leading to a permanent magnetism in the sample. In addition to that the mixture of superparamagnetic and blocked superparamagnetic NPs can explain the extremely low remanence ratios for all the samples.

Finally it is worth noting that a similar study with Au NPs shows the onset of ferromagnetism only in very small (below $2 \mathrm{~nm}$ in diameter) thiol derivatized NPs. ${ }^{8}$ For surfactant protected Au NPs only a diamagnetic behavior was measured presumably because Pd is closer to satisfying the Stoner criterion than $\mathrm{Au}$.

\section{CONCLUSIONS}

Monodisperse and stable Pd NPs have been prepared through two different chemical methods: protecting the metal NP with an alkyl ammonium salt or promoting the capping with an alkanethiol chain. Modifying the experimental conditions, NPs with different microstructure and sizes are obtained. The microstructural analysis reveals the formation of a depletion shell of Pd bonded to $\mathrm{S}$, surrounding the metal core in the thiol capped Pd NPs. In the case of alkylammonium protected NPs when the chain length is excessively large to achieve an effective protection of the metal cluster, the formation of an oxidized Pd overlayer at the surface has been also found. For Pd NPs protected with a short chainlength surfactant, the Pd clusters are more likely the pure metallic palladium clusters.

The origin of the magnetic behavior found for all these Pd NPs is different depending on their sizes and structural features. Two different mechanisms have been proposed in this paper to explain the experimentally observed differences in magnetic behavior. Metal clusters around $2 \mathrm{~nm}$ in size, without covalent links to protective molecules and without oxide passivation layer, are likely pure metallic Pd clusters and are able to stabilize those effects increasing the density of states near the Fermi level giving place to a ferromagnetic order: Surface anisotropy and twin boundaries. When a depletion shell structure is promoted, the atoms depleting the NP (S or O) are able to increase the $4 \mathrm{~d}$ density of holes at the Pd site and to localize a magnetic moment, leading to a permanent magnetism in the sample. This phenomenon is observed for a large temperature range when the depletion layer is formed by $\mathrm{Pd}-\mathrm{S}$ bonds.

\section{ACKNOWLEDGMENTS}

XAS facilities at BM29 in ESRF and the technical support from O. Mathon are acknowledged. The authors thank I. Rosa for technical assistance in sample preparation. Financial support from the Spanish MCyT and "Junta de Andalucía" is also acknowledged.
*Present address: Departamento de Física de la Materia Condensada, Universidad de Cádiz, 11510 Puerto Real, Cádiz, Spain.

${ }^{1}$ B. Sampedro, P. Crespo, A. Hernando, R. Litrán, J. C. SánchezLópez, C. López-Cartes, A. Fernández, J. Ramírez, J. GonzálezCalbet, and M. Vallet, Phys. Rev. Lett. 91, 237203 (2003).

${ }^{2}$ V. Kumar and Y. Kawazoe, Eur. Phys. J. D 24, 81 (2003).

${ }^{3}$ N. Takano, T. Kai, K. Shiiki, and F. Terasaki, Solid State Commun. 97, 153 (1996).

${ }^{4}$ T. Taniyama, E. Ohta, and T. Sato, Europhys. Lett. 38, 195 (1997).

${ }^{5}$ T. Shinoara, T. Sato, and T. Taniyama, Phys. Rev. Lett. 91, 197201 (2003).

${ }^{6}$ A. J. Cox, J. G. Louderback, S. E. Aspel, and L. A. Bloomfield, Phys. Rev. B 49, 12295 (1994).

${ }^{7}$ E. Hüger and K. Osuch, Europhys. Lett. 63, 90 (2003).

${ }^{8}$ P. Crespo, R. Litrán, T. C. Rojas, M. Mutigner, J. M. de la Fuente, J. C. Sánchez-López, M. A. García, A. Hernando, S. Penadés, and A. Fernández, Phys. Rev. Lett. 93, 087204 (2004).

${ }^{9}$ M. T. Reetz and M. Maase, Adv. Mater. (Weinheim, Ger.) 11, 773 (1999).

${ }^{10}$ M. Brust, M. Walker, D. Bethell, D. Schiffrin, and R. Whyman, J. Chem. Soc., Chem. Commun. 1994, 801.
${ }^{11}$ C. M. Shen, Y. K. Su, H. T. Yang, and H. J. Gao, Chem. Phys. Lett. 373, 39 (2003).

${ }^{12}$ D. Bonin, P. Kaiser, C. Freitigny, and J. Desbarres, in Structures Fines d'e Absorption des Rayos X en Chimie, 3, Logiciels d' Analyse, EXAFS, edited by H. Dexpert, A. Michalowizc, and M. Verdager (Societé Francaise de Chimie, Paris, 1989).

${ }^{13}$ S. I. Zabinsky, J. J. Rehr, A. Ankudinov, R. C. Albers, and M. J. Eller, Phys. Rev. B 52, 2995 (1995).

${ }^{14}$ H. Murayama, N. Ichikuni, Y. Negishi, T. Nagata, and T. Tsukuda, Chem. Phys. Lett. 376, 26 (2003).

${ }^{15}$ C. López-Cartés, T. C. Rojas, R. Litrán, D. Martínez-Martínez, J. M. de la Fuente, S. Penadés, and A. Fernández, J. Phys. Chem. B 109, 8761 (2005).

${ }^{16}$ Y. Negishi, H. Murayama, and T. Tsukuda, Chem. Phys. Lett. 366, 561 (2002).

${ }^{17}$ H. Modrow, S. Bucher, J. Hornes, R. Brinkmann, and H. Bönneman, J. Phys. Chem. B 107, 3684 (2004).

${ }^{18}$ M. Venkatesan, C. B. Fitzgerald, J. G. Lunney, and J. M. D. Coey, Phys. Rev. Lett. 93, 177206 (2004).

${ }^{19}$ P. Gambardella, S. Rusponi, M. Veronese, S. S. Dhesi, C. Grazioli, A. Dallmyer, I. Cabria, R. Zeller, P. H. Dederichs, K. Kern, C. Carbone, and H. Brune, Science 300, 1130 (2003). 